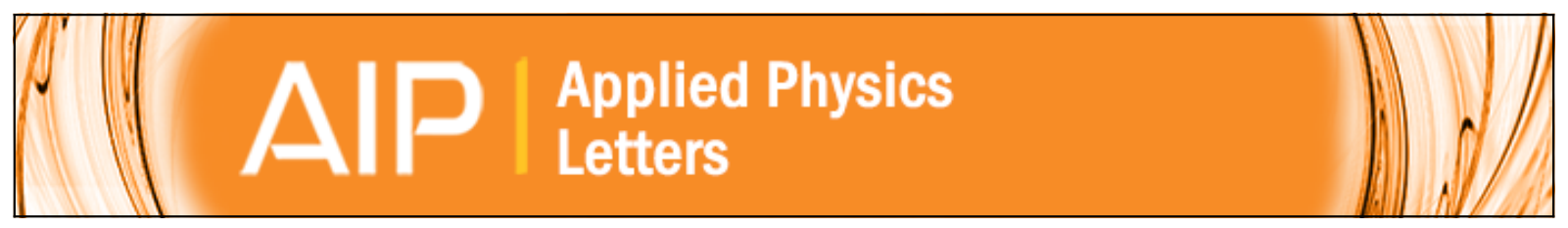

\title{
Scaling laws for jet pulsations associated with high-resolution electrohydrodynamic printing
}

Hong Kyoon Choi, Jang-Ung Park, O Ok Park, Placid M. Ferreira, John G. Georgiadis, and John A. Rogers

Citation: Applied Physics Letters 92, 123109 (2008); doi: 10.1063/1.2903700

View online: http://dx.doi.org/10.1063/1.2903700

View Table of Contents: http://scitation.aip.org/content/aip/journal/apl/92/12?ver=pdfcov

Published by the AIP Publishing

\section{Articles you may be interested in}

Electrospray pulsation: A diagnostic to understand cone-jet stability and minimum flow

J. Appl. Phys. 115, 044905 (2014); 10.1063/1.4862805

Electrohydrodynamic printing under applied pole-type nozzle configuration

Appl. Phys. Lett. 102, 024101 (2013); 10.1063/1.4775672

Onset condition of pulsating cone-jet mode of electrohydrodynamic jetting for plane, hole, and pin type electrodes J. Appl. Phys. 108, 102804 (2010); 10.1063/1.3511685

Design and evaluation of a silicon based multi-nozzle for addressable jetting using a controlled flow rate in electrohydrodynamic jet printing

Appl. Phys. Lett. 93, 243114 (2008); 10.1063/1.3049609

Scaling laws for pulsed electrohydrodynamic drop formation

Appl. Phys. Lett. 89, 124103 (2006); 10.1063/1.2356891

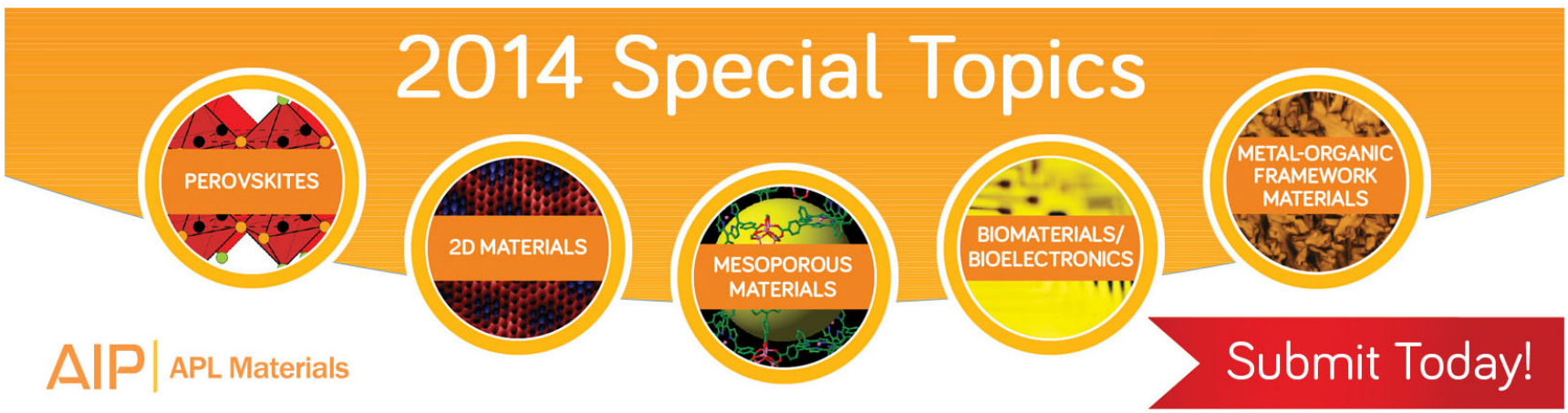




\title{
Scaling laws for jet pulsations associated with high-resolution electrohydrodynamic printing
}

\author{
Hong Kyoon Choi, ${ }^{1}$ Jang-Ung Park, ${ }^{2}$ O Ok Park, ${ }^{1, a)}$ Placid M. Ferreira, ${ }^{3}$ \\ John G. Georgiadis, ${ }^{3}$ and John A. Rogers ${ }^{2, b)}$ \\ ${ }^{1}$ Department of Chemical and Biomedical Engineering, BK21 Graduate Program of KAIST, Korea \\ Advanced Institute of Science and Technology, Daejeon 305-701, Republic of Korea \\ ${ }^{2}$ Department of Material Science and Engineering, Beckman Institute and Frederick Seitz. Materials \\ Research Laboratory, University of Illinois at Urbana-Champaign, Urbana, Illinois 61801 USA \\ ${ }^{3}$ Department of Mechanical Science and Engineering, University of Illinois at Urbana-Champaign, Urbana, \\ Illinois 61801 USA
}

(Received 5 December 2007; accepted 8 March 2008; published online 27 March 2008)

\begin{abstract}
This paper presents simple scaling laws that describe the intrinsic pulsation of a liquid jet that forms at the tips of fine nozzles under electrohydrodynamically induced flows. The jet diameter is proportional to the square root of the nozzle size and inversely proportional to the electric field strength. The fundamental pulsation frequency is proportional to the electric field strength raised to the power of 1.5. These scaling relationships are confirmed by experiments presented here and by data from the literature. The results are important for recently developed high-resolution ink jet printing techniques and other applications using electrohydrodynamics. (C) 2008 American Institute
\end{abstract} of Physics. [DOI: 10.1063/1.2903700]

Electric field induced formation of micron and nanometer sized droplets is useful in a number of different fields including electrospray mass spectroscopy ${ }^{1}$ and processing of biomaterials, ${ }^{2}$ electrohydrodynamic atomization, ${ }^{3}$ and other applications. Similar physics, particularly when used to induce pulsating jets as opposed to steady cone jets, ${ }^{4,5}$ can be exploited for printing liquid inks, with the possibility for resolution that extends into the submicron range, ${ }^{6}$ orders of magnitude better than that available from commercial thermal or piezoelectric inkjet printers. ${ }^{7,8}$ Figure 1 shows a schematic illustration of the nozzle and substrate in this electrohydrodynamic jet (e-jet) printing technique, ${ }^{6}$ together with the simulated static electric field distribution and an example of printing of dots with $\sim 1.5 \mu \mathrm{m}$ diameters. The robust operation of printers of this type, which use pulsating jets, requires delicate control of the jetting frequencies together with ultrafine (microns to fractions of a micron in inner diameter) nozzles. This paper presents scaling laws for this process, with comparison to experimental results.

The experiments were performed using a test setup similar to the printer of Fig. 1(a), but without the full computer coordinated control systems and staging apparatus needed for printing. This measurement setup was mounted under an optical microscope with a high-speed camera (Phantom v7.0, Vision Research). The dynamic motions of the meniscus at the nozzle tip under the given dc voltages were recorded to analyze the jetting frequency. A manual linear stage controlled the standoff height (typically 100-300 $\mu \mathrm{m}$ ) between the nozzle tip and a gold-coated silicon wafer (substrate) and a motorized linear stage (T-LA60A, Zaber Technologies Inc.) translated the substrate. The nozzles consisted either of goldcoated glass microcapillaries (inner diameters: 2, 10, and $30 \mu \mathrm{m}$, World Precision Instruments) or of metal capillaries (inner diameter: $100 \mu \mathrm{m}$, EFD Inc.). Compressed air that passed through a pressure regulator (type 800, ControlAir,

\footnotetext{
${ }^{\text {a)}}$ Electronic mail: ookpark@kaist.ac.kr.

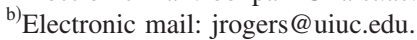

Inc.) delivered the ink to the nozzle tip through the application of a constant pressure. We configured the nozzle to eject droplets in a horizontal direction toward a substrate that vertically stands for easy observation through an optical microscope. This setup is different than the printer where the nozzle points downward and the substrate is horizontal. We found that the force of gravity is negligible compared to the other forces (e.g., electrical, surface tension) in both horizontal and vertical nozzle orientations. A dc voltage was applied between the substrate and the nozzle to create an electric field distribution [Fig. 1(b)] that induced electrohydrodynamic flows. Two different solutions were printed: (i) water $(0.1 \mathrm{mM} \mathrm{KBr}$ added) and (ii) glycerine. Jetting frequencies were measured from images of the meniscus, the jets, and droplets on the substrate, using a high-speed camera connected to the microscope. In a typical experiment, we observed that with increasing voltage, the meniscus deformed to a classic Taylor cone shape. ${ }^{9,10}$ At sufficiently high voltages, a jet emerged from the tip of the conical meniscus. ${ }^{11}$ This jet moved toward the substrate where it impinged on the surface to accumulate a droplet. After breaking, the jet recoiled back to the nozzle, leaving a printed droplet behind. $^{5,12}$

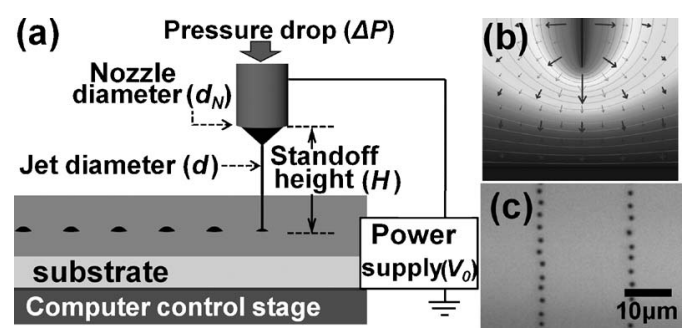

FIG. 1. (a) Schematic illustration of the nozzle and substrate in e-jet printing, with identification of key parameters used in the scaling analyses. (b) Computed equipotential lines and electric field vectors (arrows) between the nozzle and a flat plate. (c) E-jet printed arrays of $\sim 1.5 \mu \mathrm{m}$ dots formed using a nozzle with a $2 \mu \mathrm{m}$ inner diameter. (Ink: 9:1 $(v / v)$ water and glycerine mixture with $0.1 M \mathrm{NaCl}$.) 


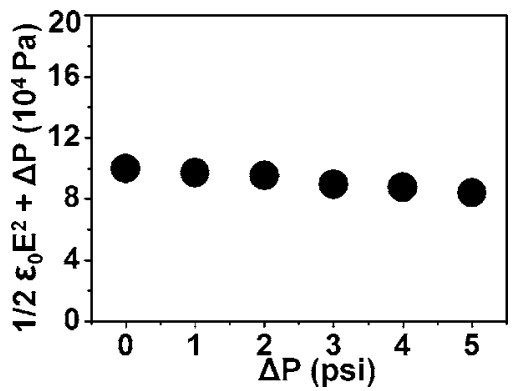

FIG. 2. Sum of electrical stress $\left(\frac{1}{2} \varepsilon_{0} E^{2}\right)$ and pressure drop $(\Delta P)$ at the condition for initiation of jetting as a function of pressure drop $(\Delta P)$. The standoff height $(H)$ was $100 \mu \mathrm{m}$ and the nozzle had a $2 \mu \mathrm{m}$ inner diameter.

The overall flow rate is an important parameter in e-jet printing; it involves the balance of electrical stress, capillary pressure, and applied pressure. Chen et al. suggested a Poiseuille-type flow rate relation for this type of system, ${ }^{13,14}$ according to

$$
Q \approx \frac{\pi d_{N}^{4}}{128 \mu L}\left(\Delta P+\frac{1}{2} \varepsilon_{0} E^{2}-\frac{4 \gamma}{d_{N}}\right) .
$$

In Eq. (1), $Q$ is the flow rate, $\Delta P$ is the pressure drop, $\mu$ is the viscosity of the liquid, $d_{N}$ and $L$ are the diameter and length of the nozzle, respectively, $\varepsilon_{0}$ is permittivity of free space, $\gamma$ is the surface tension of the air-ink interface, and $E$ is the magnitude of the electric field. We use Eq. (1) to establish scaling relationships and the approximate relative magnitudes of key parameters. We approximate $E$ at the tip of the nozzle using a model of a semi-infinite wire perpendicular to an infinite planar counter electrode, according to $E=4 V_{0} /\left[d_{N} \ln \left(8 H / d_{N}\right)\right] \cdot{ }^{15,16}$ where $H$ and $V_{0}$ are the standoff height and the imposed potential between nozzle and substrate, respectively.

Upon gradually raising $V_{0}$ from $0 \mathrm{~V}$, one observes that fluid begins to flow from the nozzle at a certain minimum voltage. This voltage approximately corresponds to stress balance in Eq. (1), when the sum of $\Delta P$ and $\frac{1}{2} \varepsilon_{0} E^{2}$ exceeds the capillary pressure $\left(4 \gamma / d_{N}\right)$. We refer to this situation as the "condition for initiation of jetting." The stress balance in Eq. (1) approximately described this initiation condition. Figure 2 shows that the sum of $\frac{1}{2} \varepsilon_{0} E^{2}$ and $\Delta P$ at the condition for initiation of jetting is nearly constant at a level that corresponds to $\sim 70 \%$ of $4 \gamma / d_{N}$ when the literature value for the surface tension of glycerine is used $(63 \mathrm{dyn} / \mathrm{cm}$ under no electric field at room temperature). This level of agreement is reasonable, given the approximate nature of the analysis and the uncertainties in the experimental measurements.

The jetting at the apex of the Taylor cone can be explained as a competition between surface tension and electric field forces. When the electric field exceeds a certain critical value, the jetting begins at the tip of the conical meniscus. Due to this physics, it is customary to define an electrical capillary number $(\mathrm{Ca})$ as a ratio between the surface tension and the electric field forces according to ${ }^{17}$

$$
\mathrm{Ca}=\frac{\varepsilon_{0}\left(E l_{2}\right)^{2}}{\gamma l_{1}},
$$

where $l_{1}$ and $l_{2}$ are characteristic length scales associated with the surface tension force and the electrical field force, respectively. We choose the diameter of the jet $d$ for $l_{2}$ and $d_{N}$ for $l_{1}$ because the surface tension acts on the entire area

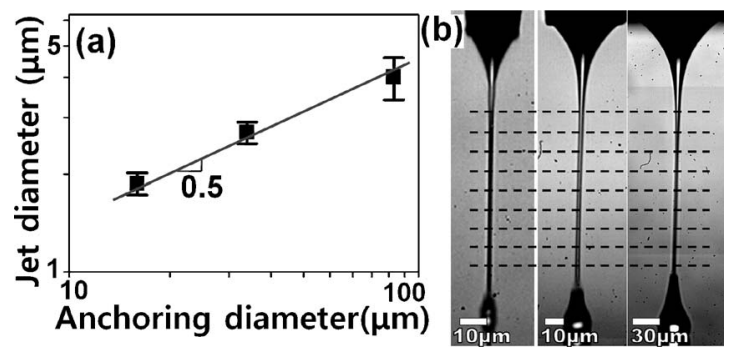

FIG. 3. (a) Jet diameter $(d)$ as a function of anchoring diameter $\left(d_{N}\right)$ at the condition for initiation of jetting. The slope of the data in this log-log plot is $\sim 0.5$, consistent with the prediction of Eq. (3). (b) Images collected with a high-speed camera, captured during jetting images. A glycerine ink was used. The exposure time for the images was $\sim 95 \mu \mathrm{s}$.

defined by the nozzle, while the electric field mainly focuses at the apex of the cone. ${ }^{18,19}$ The quantity $d_{N}$ can correspond to an anchoring diameter at the nozzle tip. With these assumptions, $d$ can be written as

$$
d \propto \sqrt{\frac{\gamma}{\varepsilon_{0}}} \frac{\sqrt{d_{N}}}{E} .
$$

To test the dependence of $d$ on $d_{N}$, we measured $d$ at the condition for initiation of jetting using a high-speed camera. Since $d$ is also time variant, we compared $d$ values at the time when the jet is widest, as determined by high-speed imaging. Here, the $d$ values were averaged at nine different distances from each nozzle tip (indicated as dashed lines), as shown in Fig. 3(b). This dependence of $d$ on $d_{N}$ predicted by Eq. (3) is consistent with measurements for three different nozzle diameters, as shown in Fig. 3(a). The predicted dependence of $d$ on $E$ is consistent with on literature data ${ }^{20}$ which reports that $d$ linearly decreases with increasing $V_{0}$ (proportional to $E$ ).

Marginean et al. reported that the high frequency regime is closely related with capillary waves on the surface of a charged droplet. ${ }^{21}$ From the capillary wave equation, they suggested the following scaling law between the pulsation frequency $f$ and the anchoring radius $r$ :

$$
f^{2} \approx \frac{2}{\pi^{2}} \frac{\gamma}{\rho r^{3}},
$$

which corresponds to the lowest excitation mode of a spherical droplet with negligible amount of charge. Here, $\rho$ is the density. From Eq. (4), we can derive another scaling law by using simple dimensional analysis. In most cases, $r$ corresponds best with the jet radius $d / 2$ since the pulsating jet occurs at the tip of the cone which has a scale that is comparable to $d / 2$. The surface tension force term in Eq. (4) can be replaced with an electrical force term because the jet in this case arises from an instability that roughly occurs when the surface tension force on nozzle $\gamma d_{N}$ is comparable to the electric force acting on the jet $\varepsilon_{0}(E d)^{2}$. This assumption, combined with Eq. (3), yields the following scaling law:

$$
f \propto\left(\frac{\varepsilon_{0}^{3}}{\rho^{2} \gamma}\right)^{1 / 4} \frac{E^{3 / 2}}{d_{N}^{3 / 4}} .
$$

Figure 4(a) presents a plot of $f$ versus $E$ in $\log$-log scale. The range of the observed $f$ values corresponds to the maximum that can be easily observed experimentally. We plot $E / d_{N}^{1 / 2}$ instead of $E$ to compensate for differences associated with nozzle size. Four different sets of data from the litera- 


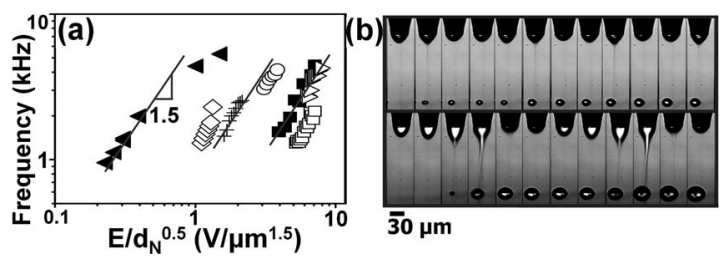

FIG. 4. (a) Pulsation frequency as a function of scaled elecric field. The slope of the data in this log-log plot is approximately $\sim 1.5$, consistent with the prediction of Eq. (5). Four different data sets were used here. ( $\square$ ) Data from Marginean et al. (Ref. 21), six different $d_{N}$, ink: $1: 1(v / v)$ methanol water mixture. $(\bigcirc,+, \diamond)$ Data from Juraschek et al. (Ref. 1), $d_{N}=60 \mu \mathrm{m}$,

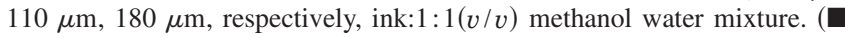
Data from Chen et al. (Ref. 13), $d_{N}=50 \mu \mathrm{m}$ ink: water, zero pressure drop applied. $(\triangleright, \square)$ Experimental data., $d_{N}=30 \mu \mathrm{m}$, ink: water with $0.1 \mathrm{mM}$ $\mathrm{KBr}$ added, $\Delta P=0$ and $\Delta P=0.5$ psi. (b) Images captured using a high-speed camera for these experiments. The time separation between adjacent images is $100 \mu \mathrm{s}$ and the exposure times were $95 \mu \mathrm{s}$. $H$ was $150 \mu \mathrm{m}$ and $E$ was $36.3 \mathrm{~V} / \mu \mathrm{m}$ for both cases. $\Delta P=0 \mathrm{psi}$ for top row and $\Delta P=0.5$ psi for bottom row.

ture and our experiments are used here. The overall trends from different experimental conditions show an approximate $\sim 1.5$ power dependence, consistent with Eq. (5). Some deviations can, however, be observed. The variation in position along the $x$ axis is likely related to differences in surface tension and pressure driven flow, as described in Eq. (1). Values of $E$ in data from Marginean et $a .^{21}$ and Juraschek and Rollgen ${ }^{1}$ are lower than those from Chen et al., ${ }^{13}$ likely due to the use of pressure driven flow and low surface tension ink (1:1 methanol/water mixture) in the former cases. Our experiments also directly reveal the effects of pressure drop. Figure 4(b) shows results that correspond to a $30 \mu \mathrm{m}$ nozzle with 0.0 and 0.5 psi pressure drop. The low-pressure drop jetting case requires higher electric fields, which exhibits higher frequencies and involves smaller droplets compared to the high pressure case. This conclusion and other aspects of the scaling behavior described here can be important in the design of systems for high-resolution e-jet printing. ${ }^{6}$
The authors acknowledge the nano-CEMMS Center at University of Illinois, which is funded by NSF under Grant No. DMI-0328162. H. K. Choi acknowledges the BK21 overseas education program from Korea. H. K. Choi and J.-U. Park equally contributed to this work.

${ }^{1}$ R. Juraschek and F. W. Rollgen, Int. J. Mass. Spectrom. 177, 1 (1998).

${ }^{2}$ V. N. Morozov and T. Y. Morozova, Anal. Chem. 71, 3110 (1999).

${ }^{3}$ R. P. A. Hartman, D. J. Brunner, D. M. A. Camelot, J. C. M. Marijnissen, and B. Scarlett, J. Aerosol Sci. 31, 65 (2000).

${ }^{4}$ K. Tang and A. Gomez, J. Colloid Interface Sci. 184, 500 (1996).

${ }^{5}$ M. Cloupeau and B. Prunet-Foch, J. Aerosol Sci. 25, 1021 (1994).

${ }^{6}$ J.-U. Park, M. Hardy, S. J. Kang, K. Barton, K. Adair, D. K. Mukhopadhyay, C. Y. Lee, M. S. Strano, A. G. Alleyne, J. G. Georgiadis, P. M. Ferreira, and J. A. Rogers, Nat. Mater. 6, 782 (2007).

${ }^{7}$ R. Parashkov, E. Becker, T. Riedl, H.-H. Johannes, and W. Kowalsky, Proc. IEEE 93, 1321 (2005).

${ }^{8}$ E. Menard, M. A. Meitl, Y. Sun, J.-U. Park, D. Shir, Y. S. Nam, S. Jeon, and J. A. Rogers, Chem. Rev. (Washington, D.C.) 107, 1117 (2007).

${ }^{9}$ G. I. Taylor, Proc. R. Soc. London, Ser. A 313, 453 (1969).

${ }^{10}$ A. Barrero, A. M. Gañán-Calvo, J. Dávila, A. Palacios, and E. Gómez-González, J. Electrost. 47, 13 (1999).

${ }^{11}$ J. F. de la Mora and I. G. Loscertales, J. Fluid Mech. 260, 155 (1994).

${ }^{12}$ This printing behavior is different than that in conventional thermal or piezoelectric drop-on demand (DOD) systems. In particular, in the method described in this paper, droplets emerge with a characteristic frequency that is not directly controlled, but which instead depends on various parameters of the system. In DOD printing, the time between successive drops can be arbitrarily defined by the user.

${ }^{13}$ C.-H. Chen, D. A. Saville, and I. A. Aksay, Appl. Phys. Lett. 89, 124103 (2006).

${ }^{14}$ According to Young-Laplace equation, capillary pressure in a tube $\Delta p$ is $2 \gamma / R$ where $R$ is radius of curvature. When the meniscus shape is a hemisphere, $\Delta p$ is $4 \gamma / d_{N}$ where $d_{N}$ is capillary inner diameter.

${ }^{15}$ C. F. Eyring, S. S. Mackeown, and R. A. Millikan, Phys. Rev. 31, 900 (1928).

${ }^{16}$ I. Marginean, P. Nemes, and A. Vertes, Phys. Rev. Lett. 97, 064502 (2006).

${ }^{17}$ G. R. Yi, J. H. Moon, and S.-M. Yang, Adv. Mater. (Weinheim, Ger.) 13, 1185 (2001).

${ }^{18}$ M. T. Harris and O. A. Basaran, J. Colloid Interface Sci. 161, 389 (1993).

${ }^{19}$ R. T. Collins, J. J. Jones, M. T. Harris, and O. S. Basaran, Nat. Phys. 4, 149 (2008).

${ }^{20}$ S. N. Jayasinghe and M. J. Edirisinghe, Appl. Phys. A: Mater. Sci. Process. 80, 399 (2005).

${ }^{21}$ I. Marginean, P. Nemes, L. Parvin, and A. Vertes, Appl. Phys. Lett. 89, 064104 (2006). 